\title{
Extraction of cocoa butter by supercritical carbon dioxide: optimization of operating conditions and effect of particle size.
}

\begin{abstract}
The optimum operating conditions for the extraction of cocoa butter from cocoa liquor using supercritical carbon dioxide and the effect of sample particle size on cocoa butter extraction under optimized operating conditions were investigated. The optimization was conducted at 10-45 MPa and 35-75C, with extraction times of 1-12 $\mathrm{h}$ by response surface methodology. The effect of particle size was studied using cocoa liquor, ground cocoa nibs and crushed cocoa nibs with particle sizes of approximately $74 \mu \mathrm{m}, 0.85-1 \mathrm{~mm}$ and $4-6 \mathrm{~mm}$, respectively. The yield was analyzed for total fat content by gravimetric method and triacylglycerol (TAG) profile by high-performance liquid chromatography. The results showed higher yield of cocoa butter with higher values of pressure, temperature and extraction time. The optimum conditions for cocoa butter extraction were $45 \mathrm{MPa}, 75 \mathrm{C}$ and $12 \mathrm{~h}$. The smaller particle size produced a higher yield of cocoa butter. 1,3-Dipalmitoyl-2-oleoyl-glycerol (POP), 1palmitoyl-2-oleoyl-3-stearoyl-glycerol (POS) and 1,3-distearoyl-2-oleoyl-glycerol (SOS) were the major TAGs present in the extracted cocoa butter, with POS being the highest $(>30 \%)$ for all treatments studied.
\end{abstract}

